\title{
Isocyanate: Arbeitsbedingte Expositionen und Krankheitsbilder
}

\section{Baur}

Isocyanates: Occupational Exposures and Disorders

\section{Zusammenfassung}

Di- und Poly-Isocyanate werden in großem Umfang für die Herstellung verschiedenster Schaumstoffe, Elastomere, als Kleber, Lackhärter, Beschichtungsmaterial u.v.a.m. eingesetzt. Ihre unsachgemäße Anwendung führt zu einer gesundheitsrelevanten inhalativen, z.T. auch kutanen Aufnahme. Expositionen gegenüber Isocyanaten finden vorwiegend in der Kraftfahrzeugindustrie, im Maschinenbau, in Gießereien, im Baugewerbe und im Bergbau, in der Elektro- und Elektronikindustrie, Farbenherstellung und -anwendung, Kunststoffindustrie, Druckindustrie, Timber-, Möbel- und Textilindustrie und teilweise auch im medizinischen Bereich (z.B. Polyurethangips) statt. Gesundheitsgefährdende Belastungen durch thermische Zersetzungsprodukte von Isocyanatprodukten (Polyurethanen) und anderen Materialien werden bisher zu wenig beachtet. Unter den Gesundheitsstörungen stehen obstruktive Atemwegserkrankungen ganz im Vordergrund. Wesentlich seltener treten exogen allergische Alveolitiden und Hautekzeme auf. Eine konsequente und qualifizierte betriebsärztliche Vorsorge trägt in Ergänzung zur am gesetzlichen Regelwerk orientierten Primärprävention dazu bei, dass Erkrankungen weitestgehend vermieden werden können. Hierzu gehört auch die Etablierung eines validierten Biomonitorings gefährdeter Arbeitnehmer.

\section{Abstract}

Isocyanates are extensively used for the production of different foams and elastomers. They also serve as glues, lacquer hardeners, inks, adhesives, fillers, finishes, sealants, coating and insulation materials. Usually, their application results in inhalative, partly also in cutaneous uptake. This review describes occupational exposures to isocyanates as well as hazardous effects. Isocyanates are used in the automautive/mechanical engeneering/building and construction/mining/casting/electricity and electronic/plastics/printing/timber and furniture/white goods and textile industry, partly also in medicine. Hazardous exposures to thermal degradation products of isocyanate-based polyurethanes and other materials have also be taken into consideration. Obstructive airway diseases are the major disorder caused by isocyanates. Rare cases suffer from extrinsic allergic alveolitis or eczema. In addition to regulation-based primary prevention qualitative medical surveillance mostly prevents disorders. There is also a need for the establishment of a validated biomonitoring of endangered employees. 
Hintergrund

Isocyanate sind hoch reaktive Syntheseausgangsstoffe, deren Weltproduktion im Bereich von 5 Millionen Tonnen jährlich liegt. Sie sind gekennzeichnet durch die N=C = O-Gruppe (Tab. 1; Abb. 1).

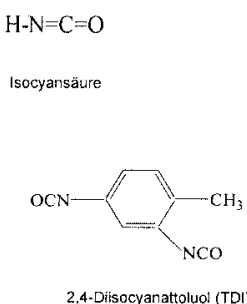

$$
\mathrm{OCN}-\left(\mathrm{CH}_{2}\right)_{6}-\mathrm{N}, \mathrm{CO}-\mathrm{NH}-\left(\mathrm{CH}_{2}\right)_{6}-\mathrm{NCO}
$$$$
\text { Aliphatisches Polyisocyanat (HDi-Biuret) }
$$

Fig. 1 Chemische Struktur wichtiger Isocyanate

Isocyanate zählen weltweit zu den häufigsten Auslösern des Berufsasthmas $[23,34,43]$. In Deutschland werden jährlich ca. 120 Anzeigen wegen des begründeten Verdachts auf eine Isocyanatbedingte Berufskrankheit (BK-Nr. 1315) erstattet, gut 60 kommen zur Anerkennung. Die Dunkelziffer lässt sich nicht abschätzen.

\section{Industrielle Verwendung von Isocyanaten} und deren unbeabsichtigte Generierung

Isocyanate finden heute vielseitige Anwendung in der Industrie und im Handwerk. Methylisocyanat (MIC) dient als Ausgangssubstanz für Insektizide. Das Trimere (Biuret) des aliphatischen Hexamethylen-Diisocyanats verwendet man vorwiegend als Lackhärter, die aromatischen Verbindungen Diisocyanattoluol (TDI), Diphenylmethandiisocyanat (MDI) und dessen Polymere für die Herstellung verschiedener Schäume, thermoplastischer Polyurethane, als Kleber in der Gesteinsverfestigung und Gusskernfertigung, beim Schmelzkleben und Folienkaschieren. Durch gezielte Auswahl der Isocyanate und deren Reaktionspartner, der Polyole, sowie genau festgelegter Reaktionsbedingungen lässt sich eine sehr breite Palette von Produkten erzeugen, die die unterschiedlichsten Materialeigenschaften aufweisen. Da Polyurethane thermisch nicht stabil sind, bilden sich unter Erhitzen (über $150^{\circ} \mathrm{C}$ ) und beim Verbrennen Isocyanate zurück bzw. neu (s.u.)

\section{Wann treten Isocyanat-Expositionen auf?}

In Deutschland haben ca. 50.000 Arbeitnehmer regelmäßig Kontakt mit Isocyanaten. Im Vordergrund stehen Isocyanatdämpfe in der Herstellung der verschiedenartigen Polyurethan (PUR)Schäume sowie Isocyanataerosole, die v.a. im Rahmen von Lackierarbeiten auftreten. Darüber hinaus gibt es eine Vielzahl weiterer arbeitsbedingter Expositionen, v. a. in der Kraftfahrzeugindustrie, im Maschinenbau, im Baugewerbe, Tunnel- und Bergbau, in der Elektro- und Elektronikindustrie, in Lackierereien, in der Druck-, Kunststoff, Textil-, Lebensmittel- und Möbelindustrie und in der Orthopädie [52].

Zu beachten ist, dass Erhitzen von PUR-Schaum zur Rückbildung verschiedener Isocyanate führt (Tab.2). Dabei entstehen bei Temperaturen bis $350^{\circ} \mathrm{C}$ überwiegend monomere Diisocyanate, bei höheren Temperaturen hauptsächlich Monoisocyanate $[14,28]$. Polyurethanschaum auf MDI-Basis bildet dabei zu 75\% partikuläre Isocyanate, denen eine hohe Lungentoxizität zukommt. Unter Erhitzen von Polyurethanlacken auf Metallblechen auf $400^{\circ} \mathrm{C}$ wird v. a. Isocyansäure freigesetzt [15].

Beim Schweißen von MDI-PUR-ummantelten Heizungsrohren bilden sich hohe Konzentrationen von Isocyansäure (ICA), 2,4'und 4,4'-MDI, 4,4'-Methylendiphenylaminoisocyanat (MAI), andere Isocyanate und das kanzerogene Methylendiphenyldiamin (MDA) [15]. Schweißarbeiten an PKWs können kurzzeitig mit sehr hohen Gesamt-Isocyanatbelastungen verbunden sein $[14,15]$. Auch Schweißen PUR-gecoateter Bleche kann mehrfache Luftgrenzwertüberschreitungen hervorrufen, insbesondere von HDI; zusätzlich sind ICA, MIC, MAI und Hexamethylenaminoisocyanat (HAI) im Atembereich nachweisbar. Außerdem zeigen Luftanalysen von Schleif-, Schneid- und Schweißarbeiten in Autowerkstätten, dass V.a. Schneiden mit hohen Isocyanatbelastungen verbunden ist [14].

Tab. 1 Physikalisch-chemische Eigenschaften und Einstufung häufig verwendeter Isocyanate

\begin{tabular}{|c|c|c|c|c|c|c|c|c|c|}
\hline Eigenschaften & MDI & Poly-MDI & $N D I$ & 2,4-TDI & 2,6-TDI & IPDI & HDI & HDI-Biuret & MIC \\
\hline $\begin{array}{l}\text { Aggregatzustand } \\
\text { bei Raumtemperatur }\end{array}$ & fest & flüssig & fest & flüssig & flüssig & flüssig & flüssig & flüssig & flüssig \\
\hline Bruttoformel & $\mathrm{C}_{15} \mathrm{H}_{10} \mathrm{~N}_{2} \mathrm{O}_{2}$ & variabel & $\mathrm{C}_{12} \mathrm{H}_{6} \mathrm{~N}_{2} \mathrm{O}_{2}$ & $\mathrm{C}_{9} \mathrm{H}_{6} \mathrm{~N}_{2} \mathrm{O}_{2}$ & $\mathrm{C}_{9} \mathrm{H}_{6} \mathrm{~N}_{2} \mathrm{O}_{2}$ & $\mathrm{C}_{12} \mathrm{H}_{18} \mathrm{~N}_{2} \mathrm{O}_{2}$ & $\mathrm{C}_{8} \mathrm{H}_{12} \mathrm{~N}_{2} \mathrm{O}_{2}$ & $\mathrm{C}_{23} \mathrm{H}_{38} \mathrm{~N}_{6} \mathrm{O}_{5}$ & $\mathrm{C}_{2} \mathrm{H}_{3} \mathrm{NO}$ \\
\hline Schmelzpunkt $\left({ }^{\circ} \mathrm{C}\right)$ & 40 & $<5$ & 127 & 22 & 9 & $<-60$ & -67 & -19 & -45 \\
\hline $\begin{array}{l}\text { Siedepunkt }\left({ }^{\circ} \mathrm{C}\right) \text { bei } \\
(\mathrm{hPa})\end{array}$ & $208(13,3)$ & $\begin{array}{l}>260 \\
>(1013)\end{array}$ & $244(133)$ & $247(1013)$ & $121(13,3)$ & $216(133)$ & $187(133)$ & & 39 (1013) \\
\hline \multirow{2}{*}{$\begin{array}{l}\text { Luftgrenzwert (ppb; } \\
\left.\mathrm{ml} / \mathrm{m}^{3}\right)\left[\mathrm{\mu g} / \mathrm{m}^{3}\right]\end{array}$} & 5 & 5 & 10 & & & 10 & 5 & & 10 \\
\hline & {$[50]$} & & [90] & & & [90] & [35] & & {$[24]$} \\
\hline MAK-Krebskategorie & $3 B$ & $3 B$ & & $3 \mathrm{~A}$ & $3 \mathrm{~A}$ & & & & \\
\hline
\end{tabular}

Abkürzungen: $\mathrm{HDI}=$ Hexamethylendiisocyanat, IPDI = Isophorondiisocyanat, MDI = Diphenylmethandiisocyanat, MIC = Methylisocyanat, NDI = Naphthylendiisocyanat, TDI = Diisocyanattoluol, $\mathrm{ppb}=$ part per billion (Milliarde) 
Tab. 2 Luftkonzentrationen verschiedener Isocyanate im Atembereich (jeweils in $\mu \mathrm{g} / \mathrm{m}^{3}$ ) Beachte, dass die Ausgangsbedingungen wegen der verschiedenen Materialien, Mengen und Temperaturen nicht immer vergleichbar sind.

\begin{tabular}{|c|c|c|c|c|c|c|c|c|c|c|c|c|c|c|}
\hline Autobrand in Garage & 17 & 4,4 & 2,9 & & 43 & 1,4 & 34 & 6,3 & 1,1 & & 3100 & & & \\
\hline $\begin{array}{l}\text { Eisengießen in Furanharz- } \\
\text { verklebte Sandkerne }\end{array}$ & & & & & & & 30 & & & & 700 & & & \\
\hline $\begin{array}{l}\text { Steinwolle, Phenol-Formaldehyd- } \\
\text { Harnstoff-Harz beschichtet; } \\
450^{\circ} \mathrm{C}\end{array}$ & & & & & & & $\sim 8$ & & & & $\sim 50$ & & & \\
\hline Schweißen PUR-gecoateter Bleche & & & & & & 130 & 10 & & & & 110 & 20 & 10 & \\
\hline Bleche schneiden & $\sim 80$ & $\sim 20$ & $\sim 30$ & & & $\sim 7$ & $\sim 20$ & & & & & & & \\
\hline $\begin{array}{l}\text { Schweißen von PUR-ummantelten } \\
\text { Heizungsrohren }\end{array}$ & & & $\sim 1100$ & $\sim 250$ & $\sim 45$ & & $\sim 45$ & & & & $\sim 900$ & & $\sim 750$ & $\sim 120$ \\
\hline
\end{tabular}

Quellen: [14-16,51]

Abkürzungen: $\mathrm{EIC}=$ Ethylisocyanat, $\mathrm{HAI}=$ Hexamethylenaminoisocyanat, $\mathrm{HDI}=$ Hexamethylendiisocyanat, ICA = Isocyansäure, IPDI = Isophorondiisocyanat, $\mathrm{MAI}=$ Diphenylmethanaminoisocyanat, $\mathrm{MDA}=4,4$-Diaminodiphenylmethan, $\mathrm{MDI}=$ Diphenylmethandiisocyanat, $\mathrm{MIC}=$ Methylisocyanat, $\mathrm{NDI}=\mathrm{Naphthylendiiso-}$ cyanat, PHI = Phenylisocyanat, PIC = Propylisocyanat, PUR = Polyurethan, TDI = Diisocyanattoluol

Bei Autobränden im Freien findet man mittlere Konzentrationen an ICA sowie eher niedrige Konzentrationen an IPDI und MIC [16]. Ein Autobrand in der Garage geht mit hohen Konzentrationen vor allem an ICA einher; aber auch die meisten anderen Isocyanatverbindungen sind dabei festzustellen [16].

Erhitzung von oberflächenbehandelter Steinwolle auf über $300^{\circ} \mathrm{C}$ führt v.a. zur Freisetzung von ICA und von MIC. Auch beim Eisengießen in mit Furanharz geklebten Sandkernen tritt ICA in hohen Konzentrationen auf, vereinzelt auch MIC. Als Quellen sind sowohl die Harz-verklebten Sandkerne als auch die Mineralwolle anzusehen [15]. Bei der Verbrennung von Chip-Platinen auf Basis von Phenol-Formaldehyd-Harnstoff-Harz (Bakelite-Kunststoff) bilden sich hohe Konzentrationen an ICA [16].

Hervorzuheben ist, dass die Luftanalytik mit Routineverfahren wie dem Bandmonitor MDA 7100 tröpfchenförmige und partikuläre Isocyanate (entstehen v.a. bei der Abkühlung von MDI, NDI und Polyisocyanaten) kaum erfasst [30]. Von unserer Arbeitsgruppe wurden wiederholt Isocyanat-Überempfindlichkeiten bei negativen Ergebnissen der Arbeitsplatz-bezogenen Messungen festgestellt.

\section{Inkorporation und Metabolite der Isocyanate}

Eingeatmete Isocyanate reagieren innerhalb des Flüssigkeitsfilms der Atemwege hauptsächlich mit Glutathion und Albumin $[6,11,26,24,54]$. Im Blut lassen sich Albumin- und Hämoglobingebundene Isocyanat-Metabolite als (Di)Aminaddukte nachweisen. Isocyanate binden auch an oberflächlichen Strukturen des Atemwegsepithels [19]. Ein wichtiger Reaktionspartner in den Bronchien ist Keratin-18, dem als Reaktionspartner der Haut Keratin-10 entspricht. Auch im Verbund mit Tubulin, einer ProteinUntereinheit der ziliaren Mikrotubuli, wurden Addukte nachge- wiesen [25]. In-vitro-Inkubationen ergaben darüber hinaus Bindungen an 11-Dihydrodiol-dehydrogenase, an Actin und ein 78 kDa großes Glukose-reguliertes, Stress-induzierbares Protein [54].

Bemerkenswert ist die systemische Verteilung im Organismus von in hoher Dosis inhalativ aufgenommenen Isocyanaten bzw. deren Metaboliten wie tierexperimentelle Studien belegen $[20,21,41]$.

\section{Kann Hautkontalkt eine respiratorische Überempfindlichkeit auslösen?}

Erjefalt und Persson [8], Rattray u. Mitarb. [40], Låstbom u. Mitarb. [27], Karol [17] und Karol u. Mitarb. [18] induzierten im Tierexperiment durch dermale Applikationen von TDI bzw. MDI spezifische respiratorische Überempfindlichkeiten. Ein gleichartiger Mechanismus wird auch beim Menschen angenommen $[3,22,31,44,54]$. Petsonk u. Mitarb. [38] fanden entsprechende Hinweise; sie untersuchten 214 Beschäftigte in der holzverarbeitenden Industrie (Herstellung von Holzlaminaten unter Verwendung eines Klebers mit monomerem und polymerem MDI). Unter den 56 Personen, die den meisten Kontakt mit dem flüssigen Isocyanatkleber hatten, entwickelten 15 (27\%) ein neu aufgetretenes Asthma bronchiale. Keiner der 43 Arbeiter in der niedrigsten Expositionsgruppe zeigte eine derartige Erkrankung. In der ersteren Gruppe wiesen $47 \%$ jener Personen, die Isocyanat-Hautkontakt bemerkten, eine neu aufgetretene Asthmaerkrankung auf, während 19\% der Subgruppe ohne einen solchen Kontakt erkrankten. 
lisierungen sowie eine Verschlechterung der Lungenfunktion zu beobachten sind $[13,29]$.

Alle Isocyanate haben eine starke lokale Reizwirkung an den Schleimhäuten des Atemtrakts und der Augen, ferner an der Haut. Dies trifft sowohl für gasförmige, tröpfchenförmige (v.a. Lacknebel) als auch partikuläre Isocyanate zu. Daneben ist eine eher schwache sensibilisierende Wirkung sowohl tierexperimentell als auch beim Menschen belegt, die sich in Form von IgEvermittelten Sofortreaktionen (in ca. $20 \%$ der Isocyanat-Asthmatiker feststellbar) und - sehr selten - als exogen allergische Alveolitis manifestiert [2]. Klinisch stehen die akuten Reizwirkungen am Atemtrakt, die schon im Bereich der aktuellen Luftgrenzwerte unter suszeptiblen Personen festzustellen sind, ganz im Vordergrund. Etwa 5\% der Exponierten entwickeln eine obstruktive Atemwegserkrankung. Abb. 2 zeigt eine duale asthmatische Reaktion eines Patienten, der infolge einer einjährigen Exposition gegenüber MDI ein Berufsasthma entwickelte.

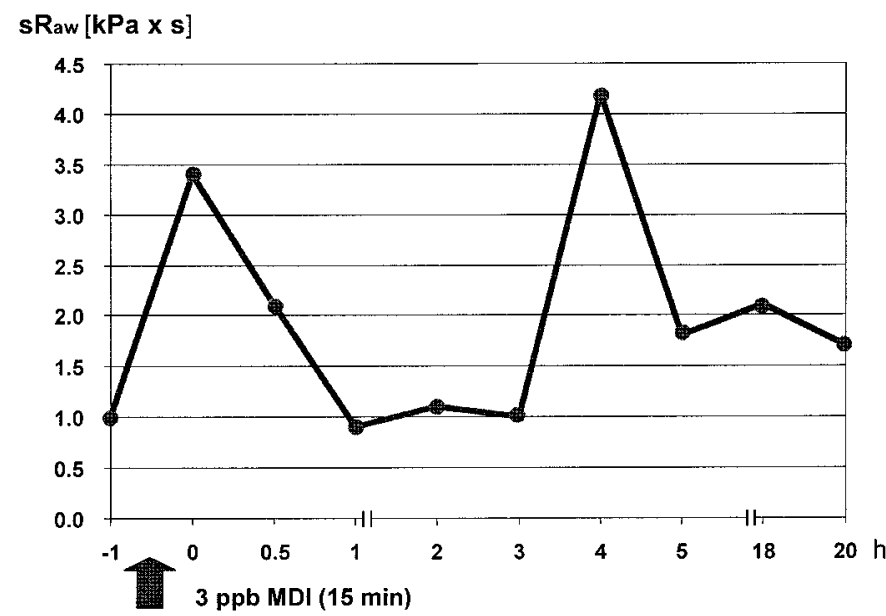

Fig. 2 Arbeitsplatzbezogener inhalativer Expositionstest mit dem Isocyanat Diphenylmethandiisocyanat (MDI).

Die Prognose des Isocyanatasthmas ist nach langjähriger Beschwerden-assoziierter Exposition ungünstig, insbesondere, wenn es sich um IgE-negative Fälle handelt [32,36,39,42]. Hervorzuheben sind in mehreren Langzeitstudien v.a. unter hochbelasteten Isocyanatarbeitern belegte Lungenfunktionseinschränkungen $[5,7,12,35,37,53]$.

Polymeres TDI, MDI und Poly-MDI zeigen in Tierversuchen kanzerogene Wirkungen [4], die aber noch keine abschließende Einstufung erlauben (aktuell Kategorie 3 A bzw. 3 B; vgl. Tab.1). Auch ergeben diesbezüglich die wenigen epidemiologischen Studien noch keine sichere Aussage $[9,10,50]$.

\section{Luftgrenzwerte und Biomonitoring}

Tab. 1 listet die aktuellen Luftgrenzwerte, die in Deutschland gelten, auf. Aus Verlaufsuntersuchungen sowie aus eigenen klinischen Erfahrungen ergeben sich Hinweise, dass zumindest die Grenzwerte für die aromatischen Isocyanate zu hoch angesetzt sind und unter entsprechenden Expositionen langfristig Sensibi-
In Deutschland gibt es im Gegensatz zu England und Schweden keine Luftgrenzwerte für TDI-Polymere, Poly-HDI und Isocyansäure, auch nicht für den Gesamtgehalt an Isocyanatgruppen. Eine entsprechende Festsetzung ist unter primärpräventiven Aspekten dringend geboten.

Für MDI- und Poly-MDI-exponierte Arbeitnehmer steht neuerdings zur Überwachung die Möglichkeit eines Biomonitorings zur Verfügung, da im Urin der Metabolit 4,4"-Diaminodiphenylmethan (MDA), nachweisbar ist (BAT-Wert $10 \mu \mathrm{g} / \mathrm{g}$ Kreatinin). Diese Untersuchung gibt Information über die Exposition während der letzten 24 Stunden; wobei allerdings eine Validierung noch aussteht. Zur Zeit werden routinemäßig einsetzbare Biomonitoringverfahren auch für andere Isocyanate entwickelt $[33,48,49,55]$. Eigene Untersuchungen belegen die Vorteile des Biomonitorings, nämlich die Miterfassung der Inkorporation der luftanalytisch schwer fassbaren Aerosole und der kutanen Aufnahme (Abb. 3).

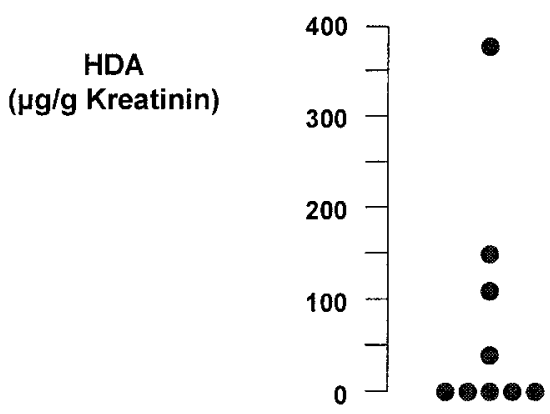

Fig. 3 Untersuchungen mit einem neuen Biomonitoring-Verfahren: 4 der 9 Spritzlackierer, die 2-Komponenten-Lacke mit HDI-Biuret-Härter anwendeten, weisen im Urin z. T. hohe Konzentrationen des Metaboliten Hexamethylendiamin (HDA) auf. Die Luftmessungen am Arbeitsplatz waren durchwegs negativ [55].

\section{Diskussion und Ausblick}

Im November 2002 fand in Norwegen ein Workshop statt, an dem eine multidisziplinäre Gruppe von über 20 unabhängigen Forschern und Experten auf dem Gebiet der Risikoerfassung und des Managements von „Isocyanatexpositionen“ teilnahmen. Das Hauptziel dieses Treffens war ein wissenschaftlich basiertes Risikomanagement zu erarbeiten, das nicht durch „ökonomische und andere spezielle Interessen und von Bias durchsetzt ist“. Es wurden der aktuelle Kenntnisstand der Gesundheitsgefährdung, deren Objektivierung und die erforderlichen Präventionsmaßnahmen eingehend diskutiert und zusammengefasst [1]. In Übereinstimmung mit dieser über das Internet zugänglichen hervorragenden Übersicht und den dort formulierten Verbesserungen des Gesundheitsschutzes ist es dringend geboten, gesundheitsbasierte Luftgrenzwerte für alle Isocyanate festzulegen und einzuhalten. Außerdem sollten die Arbeitsschutzregulatoren einschließlich der Auswahlkriterien für die arbeitsmedizinische Vorsorge die dargestellte unbeabsichtigte, aber häufige und abschätzbare Neu- bzw. Rückbildung von gasförmigen und partikulären Isocyanaten berücksichtigen. Nur so können Sensibili- 
sierungen, expositionsinduzierte Krankheitssymptome und Lungenfunktionseinschränkungen ganz überwiegend vermieden werden. Für Deutschland bedeutet dies vor allem die Berücksichtigung der niedermolekularen und der Poly-Isocyanate sowie des Gesamtisocyanatgehalts der Luft. Daneben ist eine konsequente Vermeidung des Hautkontakts in Anbetracht der starken Hinweise auf dadurch ausgelöste systemische Effekte von Bedeutung.

Auf dem norwegischen Workshop wurde, ebenso wie in mehreren Publikationen $[45-48,55]$ zu Recht auch auf die neuen, ergänzenden Überwachungsmöglichkeiten mittels des Biomonitorings hingewiesen. Dieses ist v.a. wegen der fehleranfälligen Luftanalytik von Isocyanataerosolen $[16,30]$ und der vom Aufnahmeweg unabhängigen Erfassung der inneren Dosis wichtig $[6,30]$.

Dringend erforderlich erscheinen in diesem Zusammenhang epidemiologische Langzeituntersuchungen exponierter Kohorten zur Evaluierung unbedenklicher arbeitsbedingter Belastungen sowie zur Ermittlung des nach In-vitro- und Tierversuchen anzunehmenden kanzerogenen Risikos aromatischer Isocyanate.

\section{Literatur}

${ }^{1}$ Bakke JV, Norén JO, Thorud S et al. International Consensus Report on: Isocyanates - Risk assessment and management. Gjovik: Norwegian Labour Inspection Authority, 2002 (hhtp://www.arbeidstilsynet.no/ publikasjoner/rapporter/rapport1eng.html)

2 Baur X. Occupational asthma due to isocyanates. Lung 1996; 174: $23-30$

${ }^{3}$ Beck LA, Leung DYM. Allergen sensitization through the skin induces systemic allergic responses. J Allergy Clin Immunol 2000; 106: $258-263$

${ }^{4}$ Bolognesi C, Baur X, Marczynski B et al. Carcinogenic risk of toluene diisocyanate and 4,4'-Methylenediphenyl diisocyanate: Epidemiological and experimental evidence. Crit Rev Toxicol 2001; 31: 737-767

${ }^{5}$ Clark RL, Bugler J, McDermott M et al. An epidemiology study of lung function changes of toluene diisocyanate foam workers in the United Kingdom. Int Arch Occup Environ Health 1998; 71: 169-179

${ }^{6}$ Day BW, Jin R, Basalyga DM et al. Formation, solvolysis, and transcarbamyolation reactions of bis(S-glutathionyl) adducts of 2,4- and 2,6-diisocyanatotoluene. Chem Res Toxicol 1997; 10: 424-431

${ }^{7}$ Diem JE, Jones RN, Hendrick DJ et al. Five-year longitudinal study of workers emploved in a new toluene diisocyanate manufacturing plant. Am Rev Respir Dis 1982; 126: 420-428

8 Erjefalt I, Persson CG. Increased sensitivity to toluene diisocyanate (TDI) in airways previously exposed to low doses of TDI. Clin Exp Allergy 1992; 22: $854-862$

${ }^{9}$ Hagmar L, Stromberg $\mathrm{U}$, Welinder $\mathrm{H}$ et al. Incidence of cancer and exposure to toluene diisocyanate and methylene diphenyldiisocyanate: a cohort based case-referent study in the polyurethane foam manufacturing industry. Br J Ind Med 1993; 50: $1003-1007$

${ }^{10}$ Hagmar L, Welinder H, Mikoczy Z. Cancer incidence and mortality in the Swedish polyurethane foam manufacturing industry. Br J Ind Med 1993; 50: 537-543

${ }^{11}$ Jin R, Day BW, Karo MH. Toluene diisocyanate protein adducts in the bronchoalveolar lavage of guinea pigs exposed to vapors of the chemical. Chem Res Toxicol 1993; 6: 906-912

12 Jones RN, Rando RJ, Glindmeyer HW et al. Abnormal lung function in polyurethane foam producers: weak relationship to toluene diisocyanate exposures. Am Rev Respir Dis 1992; 146: 871 - 877

${ }^{13}$ Kampen V van, Marczynski B, Baur X. Isocyanatbedingte Gesundheitsrisiken unter besonderer Berücksichtigung prepolymerer Verbindungen - eine Literaturauswertung. Zbl Arbeitsmed 1998; 48: 134-143
${ }^{14}$ Karlsson D, Spanne M, Skarping G. Airborne thermal degradation products of polyurethane coatings in car repair shops. J Environ Monit 2000; 2: $462-469$

${ }^{15}$ Karlsson D, Dalene M, Skarping G et al. Determination of isocyanic acid in air. J Environ Monit 2001; 3: 432-436

${ }^{16}$ Karlsson D, Dahlin J, Skarping G et al. Determination of isocyanates, aminoisocyanates and amines in air formed during thermal degradation of polyurethane. J Environ Monit 2002; 4: 216-222

${ }^{17} \mathrm{Karol} \mathrm{MH}$. Respiratory effects of inhaled isocyanates. Crit Rev Toxicol 1986; 16: $349-379$

${ }^{18}$ Karol MH, Hauth BA, Riley EJ et al. Dermal contact with toluene diisocyanate (TDI) produces respiratory tract hypersensitivity in guinea pigs. Toxicol Appl Pharmcol 1981; 58: $221-230$

19 Karol MH, Thorne PS, Hilebrand JA. Immunohistochemical detection of toluene diisocyanate (TDI) adducts: I. Pulmonary tissue of guinea pigs following inhalation exposure. Inhalation Toxicol 1997; 9: 63-83

${ }^{20}$ Kennedy AL, Stock MF, Alarie Y et al. Uptake and distribution of ${ }^{14} \mathrm{C}$ during and following inhalation exposure to radioactive toluene diisocyanate. Toxicol Appl Pharmacol 1989; 100: 280-292

${ }^{21}$ Kennedy AL, Singh G, Alarie Y et al. Autoradiographic analyses of guinea pig airway tissues following inhalation exposure to 14C-labeled methyl isocyanate. Fundam Appl Toxicol 1993; 20: 57-67

22 Kimber I. The role of the skin in the development of chemical respiratory hypersensitivity. Toxicol Lett 1996; 86: 89-92

${ }^{23}$ Lagier F, Cartier A, Malo J-L. Medico-legal statistics on occupational asthma in Quebec between 1986 and 1988. Rev Mal Respir 1990; 7: $337-341$

${ }^{24}$ Lange RW, Day BW, Lemus R et al. Intracellular S-glutathionyl adducts in murine lung and human bronchoepithelial cells after exposure to diisocyanatotoluene. Chem Res Toxicol 1999; 12: 931-936

${ }^{25}$ Lange RW, Lantz RC, Stolz DB et al. Toluene diisocyanate colocalizes with tubulin on cilia of differentiated human airway epithelial cell. Toxicol Sci 1999; 50: 64-71

${ }^{26}$ Lantz RC, Lemus R, Lange RW et al. Rapid reduction of intracellular glutathione in human bronchial epithelial cells exposed to occupational levels of toluene diisocyanate. Toxicol Sci 2001; 60: 348-355

${ }^{27}$ Låstbom L, Cam ner P, Ryrfeldt A. Acute lung function impairment by hexamethylene diisocyanate and toluene diisocyanate - a comparative study using isolated guinea pig lungs. Occup Hyg 1999; 2: $111-120$

${ }^{28}$ Låstbom L, Colmsjö A, Johansson R et al. Effects of thermal degradation products from polyurethane foams based on toluene diisocyanate and diphenylmethane diisocyanate on isolated, perfused lung of guinea pig. Scand J Work Environ Health 2003; 29: 152 - 158

${ }^{29}$ Latza U, Baur X, Malo J-L. Isocyanate-induced respiratory health effects. In: Bakke JV, Norén JO, S. Thorud S, Aasen TB (Hrsg.): International consensus report on: Isocyanates - Risk assessment and management, Appendices 10.2.1. Gjovik: Norwegian Labour Inspection Authority, 2002: 37-51 http://www.arbeidstilsynet.no/publikasjoner/rapporter/rapport1eng.html

${ }^{30}$ Levine SP, Hilling KJD, Dharmarajan V et al. Critical review of methods of sampling, analysis, and monitoring for TDI and MDI. Am Ind Hyg Assoc J 1995; 56: $581-589$

${ }^{31}$ Liu Y, Sparer J, Woskie SR et al. Qualitative assessment of isocyanate skin exposure in auto body shops: A pilot study. Am J Ind Med 2000; 37: $265-274$

32 Lozewisz S, Assoufi BK, Hawkins R et al. Outcome of asthma induced by isocyanates. $\mathrm{Br}$ J Dis Chest 1987; 81: $14-22$

${ }^{33}$ Lushniak BD, Reh CM, Bernstein DI et al. Indirect assessment of 4,4'-diphenylmethane diisocyanate (MDI) exposure by evaluation of specific humoral immune responses to MDFI conjugated to human serum albumin. Am J Ind Med 1998; 33: 471-477

${ }^{34} \mathrm{McDonald}$ JC, Keynes HL, Meredith SK. Reported incidence of occupational asthma in the United Kingdom, 1989-1997. Occup Environ Med 2000; 57: $823-829$

35 Omae K, Nakadate T, Nigashi T et al. Four-year follow-up of effects of toluene diisocyanate exposure on the respiratory system in polyurethane foam manufacturing workers. II. Four-year changes in the effects on the respiratory system. Int Arch Occup Environ Health 1992; 63: $565-569$

${ }^{36}$ Paggiaro PL, Loi AM, Rossi O et al. Baschiere: Follow-up study of patients with respiratory disease due to toluene diisocyanate (TDI). Clin Allergy 1984; 14: $463-469$

37 Peters JM. Cumulative pulmonary effects in workers exposed to tolylene diisocyanate. Proc R Soc Med 1970; 63: 372 - 375 
${ }^{38}$ Petsonk EL, Wang ML, Lewis DM et al. Asthma-like symptoms in wood product plant workers exposed to methylene diphenyl diisocyanate. Chest 2000; 118: 1183-1193

${ }^{39}$ Piirilä PL, Nordman H, Keskinen HM et al. Long-term follow-up of hexamethylene diisocyanate-, diphenylmethane diisocyanate-, and toluene diisocyanate-induced asthma. Am J Respir Crit Care Med 2000; 162: $516-522$

${ }^{40}$ Rattray NJ, Botham PA, Hext PM et al. Induction of respiratory hypersensitivity to diphenylmethane-4,4-diisocyanate (MDI) in guinea pigs. Influence of route of exposure. Toxicology 1994; 88: 15-30

${ }^{41}$ Redlich CA, Karol MH. Diisocyanate asthma: clinical aspects and immunopathogenesis. Int Immunopharmacol 2002; 2: 213-224

42 Rosenberg N, Garnier R, Rousselin X et al. Clinical and socioprofessional fate of isocyanate-induced asthma. Clin Allergy 1987; 17: 55-61

${ }^{43}$ Rosenman KD, Reilly MJ, Kalinowski DJ. A state-based surveillance system for work-related asthma. J Occup Environ Med 1997; 39: $415-425$

${ }^{44}$ Schröder C, Uter W, Schwanitz HJ. Occupational allergic contact dermatitis, partly airborne, due to isocyanates and epoxy resin. Contact Dermatitis 1999; 41: 117-118

${ }^{45}$ Schütze D, Sepai O, Lewalter J et al. Biomonitoring of workers exposed to $4,4^{\prime}$-methylenedianiline or $4,4^{\prime}$-methylenediphenyl diisocyanate. Carcinogenesis 1995; 16: $573-582$

${ }^{46}$ Sepai O, Henschler D, Sabbioni G. Albumin adducts, hemoglobin adducts and urinary metabolites in workers exposed to 4,4'-methylenediphenyl diisocyanate. Carcinogenesis 1995; 16: 2583-2587

47 Sepai O, Schütze D, Heinrich U et al. Hemoglobin adducts and urine metabolites of 4,4'-methylenedianiline after 4,4'methylenediphenyl diisocyanate exposure of rats. Chem Biol Interact 1995; 97: 185-198

48 Skarping G, Dalene MD, Tinnerberg H. Biological monitoring of hexamethylene- and isophorone-diisocyanate by the determination of he- xamethylene- and isophorone-diamine in hydrolysed urine using liquid chromatography and mass spectrometry. Analyst 1994; 119: $2051-2055$

${ }^{49}$ Skarping G, Dalene M, Svensson BG et al. Biomarkers of exposure, antibodies, and respiratory symptoms in workers heating polyurethane glue. Occup Environ Med 1996; 53: 187- 187

50 Sorahan R, Pope D. Mortality and cancer morbidity of production workers in the United Kingdom flexible polyurethane foam industry. $\mathrm{Br}$ J Ind Med 1993; 50: 528-536

${ }^{51}$ Bundesministerium für Arbeit und Sozialordnung. Swedish Rescue Services Agency. Working environment study. Karlstad: Räddninsverket, 2002 (ISBN 91 - 7253-157-6)

52 Bundesministerium für Arbeit und Sozialordnung. Technische Regeln für Gefahrstoffe. TRGS 430 - Isocyanate - Exposition und Überwachung. Bundesarbeitsblatt 2002; 3: 45-52

${ }^{53}$ Wegmann DH, Musk SW, Main DM et al. Accelerated loss of FEV - in polyurethane production workers: a four-year prospective study. Am J Ind Med 1982; 3: 209-215

${ }^{54}$ Wisnewski AV, Srivastava R, Herrick $C$ et al. Identification of human lung and skin proteins conjugated with hexamethylene diisocyanate in vitro und in vivo. Am J Respir Crit Care Med 2000; 162: 2330 - 2336

${ }^{55}$ Yu F, Laudehr H, Baur X et al. Ein neues Biomonitoring-Verfahren für den beruflichen Umgang mit Isocyanaten. Im Druck,

\section{Bereits publizierte Beiträge zu dieser Serie:}

${ }^{1}$ Kühlschmierstoffe. Pneumologie 2003; 57: $212-221$

${ }^{2}$ Getreidestaub. Pneumologie 2003; 57: 335-339

${ }^{3}$ Cellulasen. Pneumologie 2003; 57: 388-391 